\title{
Prediction of optical properties of $F$ centers in oxides from quasiparticle excitations
}

\author{
Sergio Tosoni, ${ }^{1,2}$ Daniel Fernandez Hevia, ${ }^{2,3}$ Jesús Perez Peña, ${ }^{2}$ and Francesc Illas ${ }^{1, *}$ \\ ${ }^{1}$ Departament de Química Física and Institut de Química Teòrica i Computacional (IQTCUB), Universitat de Barcelona, \\ C/ Martí i Franquès 1, E-08028 Barcelona, Spain \\ ${ }^{2}$ Departamento de Química, Universidad de Las Palmas de Gran Canaria, Campus Universitario de Tafira, \\ 35017 Las Palmas de Gran Canaria, Spain \\ ${ }^{3}$ INAEL Electrical Systems S.A., C/ Jarama, 5, 45007 Toledo, Spain \\ (Received 27 January 2012; published 15 March 2012)
}

\begin{abstract}
Electrons trapped at the cavity of oxygen vacancies on $\mathrm{MgO}, \mathrm{CaO}$, and $\mathrm{ZnO}$ have important physical and chemical properties. In particular they exhibit well-defined spectroscopic features. Here, these are investigated by means of the self-consistent many-body perturbation theory-based GW method, using periodic models and reasonably large supercells. The excitations predicted from the quasiparticle band structure calculations are in good agreement with experiment and with calculations from embedded cluster models, thus opening the way to interpret spectroscopic features of point defects in oxides and materials that cannot be represented by embedded clusters.
\end{abstract}

PACS number(s): 78.40.-q, 61.72.jd, 71.15.Qe

\section{INTRODUCTION}

Metal oxides are at the heart of many new and important discoveries of technological importance, such as high critical temperature superconductivity ${ }^{1}$ and colossal magnetoresistance in physics $^{2}$ and photocatalysis under visible light in chemistry. ${ }^{3}$ There is little doubt that these interesting properties originate from the very special electronic structure of these systems, and they are strongly related to their nonstoichiometry, either due to the presence of dopants or to point defects. Zinc oxide, in particular, has been of growing interest in photocatalysis and electronics, as a key material to develop protective devices in electric circuits (namely varistors). In order to fulfill the technological challenges with more demanding requisites, a new generation of materials is crucially needed, and their design may benefit from a deeper knowledge of their electronic structure-related properties. Paramount among them are those governed by excited states, which are usually described in a crude way from ground-state density of states.

In fact, despite the high relevance of providing a good description of optical properties of stoichiometric and defective oxides, a general and accurate method for the calculation of electronic excitations is still lacking. From a theoretical point of view, excited states in oxides have often been studied either by adopting a configuration-interactionembedded cluster model (CI-ECM) approach or by using different implementation of band theory from Hartree-Fock to different implementations of the density functional theory (DFT). The CI-ECM approach provides very accurate results only if the material can be represented by such local models. Periodic models are usually constrained to some form of DFT, which, for the usual standard local density approach (LDA) or generalized gradient approach (GGA) implementations of the exchange correlation, have serious problems in describing strongly correlated systems, $\mathrm{NiO}$ being prototypal. ${ }^{4,5}$ The failure of LDA and GGA is due to the self-interaction error introduced in the calculations of the Coulomb term of the potential. As a result, LDA and GGA predict a metallic character for $\mathrm{NiO}$, an experimentally well-characterized antiferromagnetic insulator. ${ }^{6}$ The same qualitatively incorrect description is found for the cuprate superconducting parent compounds and for many other materials. ${ }^{7}$ Hybrid DFT offers a remedy to this situation by mixing nonlocal Fock and Slater local exchange functionals, although at the price of introducing a certain dose of empirism. ${ }^{6-8}$ Recently developed semilocal potentials seem also to be promising. ${ }^{9}$

A possible way to overcome the problems of standard implementation of DFT in the description of the electronic structure of oxides and of other strongly correlated systems, without relying on hybrid DFT approaches, although preserving the periodic description of the solid is to make use of the GW formulation, earlier introduced by Hedin. ${ }^{10}$ The GW method goes beyond the mean-field description of the electron-electron interaction in DFT by including many-body effects in the electronic transitions. This is achieved by expanding the multielectronic problem as a series of products of one-particle Green's function $(\mathrm{G})$ and screened dielectric tensor (W). In particular, it has been shown that the $\mathrm{GW}_{0}$ approach provides quasiparticle energies, which are in good agreement with experimental excitations. ${ }^{11,12}$ A problem with the fully self-consistent $\mathrm{GW}$ calculations is that these are usually computationally very demanding. Nevertheless, recent advances in both computers and codes have made it possible to carry out these types of calculations at least for perfect bulk systems. $^{13-16}$

From the discussion above, one may conclude that the accurate prediction of the band gap of metal oxides is no longer a problem for theory. However, the situation is less clear for the description of excited states related to point defects, where the presence of the point defect breaks the periodic symmetry and introduces important features, which, in addition, may have important implications. ${ }^{17}$ In principle, the success of the various implementations of the rigorous many-body perturbation theory-based GW method commented on above opens the way for a fully periodic description of spectroscopic features related to point defects; this is precisely the goal of the present work. To this end, we investigate the absorption spectra of the experimentally well-characterized $\mathrm{F}$ centers of $\mathrm{MgO}, \mathrm{CaO}$, and $\mathrm{ZnO}$ for which accurate results arising from the CI-ECM method exists. ${ }^{18-20}$ These centers are created upon 
removing an oxygen atom or an oxygen anion $\left(\mathrm{O}^{-}\right)$from the lattice, thus leaving two ( $\mathrm{F}$ center) or one $\left(\mathrm{F}^{+}\right.$center $)$electrons trapped in the cavity, respectively. The trapped electrons are stabilized by the Madelung potential of the crystal, and their electron density appears to be well localized in the cavity formed by the oxygen vacancy. ${ }^{21}$ Thus, the trapped electrons do not occupy the empty $3 \mathrm{~s}$ levels of the neighboring $\mathrm{Mg}$ ion, and the resulting defects are better described as pseudoatoms. ${ }^{22}$ The defective materials exhibit typical excitations in the visible region of the spectrum, which thus changes its color. The name F center comes precisely from the word Farbe, which means color in German. In particular, the spectroscopic features of these point defects have been known since the original work of Chen et al. for oxygen vacancies in $\mathrm{MgO}$ in the late 1960 s. $^{23}$ In the past few years, the interest in these defects has been renewed because they have been shown to play an important role in determining the surface chemistry of these materials, ${ }^{24}$ such as acting as a nucleation center of supported clusters and defining specific nanostructures with potential technological applications. ${ }^{25}$ Clearly, an accurate description of the electronic structure of these point defects is needed.

\section{COMPUTATIONAL DETAILS}

To approach the lowest optical excitation of the $\mathrm{F}$ and $\mathrm{F}^{+}$centers of $\mathrm{MgO}, \mathrm{CaO}$, and $\mathrm{ZnO}$, suitable supercells, representing a reasonably low defect concentration, are constructed using the lattice parameter first optimized for the bulk, undefective material within the PBE implementation of the exchange-correlation functional, ${ }^{26}$ a plane-wave basis set within a cutoff of $800 \mathrm{eV}$ to describe the $\mathrm{Mg}(2 \mathrm{p}, 3 \mathrm{~s}), \mathrm{Ca}$ $(3 \mathrm{p}, 4 \mathrm{~s}), \mathrm{Zn}(3 \mathrm{~d}, 4 \mathrm{~s})$, and $\mathrm{O}(2 \mathrm{~s}, 2 \mathrm{p})$ valence electrons density, whereas the core electrons are described with the projectoraugmented-wave (PAW) method. ${ }^{27,28}$ The bulk calculations have been performed using the optimized primitive cell and sufficiently dense grids of special Monkhorst-Pack ${ }^{29} k$-points $(8 \times 8 \times 8$ for $\mathrm{MgO}$ and $\mathrm{CaO}$ and $8 \times 8 \times 4$ for $\mathrm{ZnO}$, respectively). Next, oxygen vacancies are created in a $\mathrm{M}_{27} \mathrm{O}_{27}$ $(\mathrm{M}=\mathrm{Mg}, \mathrm{Ca})$ supercell for $\mathrm{MgO}$ and $\mathrm{CaO}$ and $\mathrm{Zn}_{16} \mathrm{O}_{16}$ for $\mathrm{ZnO}$, as shown in Fig. 1; the resulting supercells are denoted as $\mathrm{M}_{27} \mathrm{O}_{26} \mathrm{O}_{\text {vac }}$ and $\mathrm{Zn}_{16} \mathrm{O}_{15} \mathrm{O}_{\text {vac }}$, respectively. For these supercells, all internal coordinates have been fully relaxed using a conjugated gradient scheme with a threshold of $0.001 \mathrm{eV} / \AA$, maintaining the lattice parameters obtained for the undefective materials. For these supercell calculations, a cutoff of $400 \mathrm{eV}$ has been employed together with a $2 \times 2 \times$ 2 for $\mathrm{MgO}$ and $\mathrm{CaO}$ and $4 \times 4 \times 2$ for $\mathrm{ZnO}$ meshes of special $k$ points.

Performing quasiparticle GW calculations as implemented in VASP ${ }^{11,13,30,31}$ requires a multistep approach. After structure relaxation, a well-converged $\mathrm{PBE}$ density is obtained with a threshold of $10^{-6} \mathrm{eV}$ for the total energy. Next, the Green quasiparticle eigenvalues are calculated within the GW approach considering at least 35 frequencies. The cutoff for the response function has been set to $150 \mathrm{eV}$. The quasiparticle calculation can be carried out non-self-consistently, by simply solving the quasiparticle equations as a first-order perturbation of the GGA eigenvalues $\left(\mathrm{G}_{0} \mathrm{~W}_{0}\right)$; self-consistently but on the Green's function eigenvalues only $\left(\mathrm{GW}_{0}\right)$ or on both the Green function and the screened dielectric matrix $(\mathrm{GW})$. In the case of the self-consistent $\mathrm{GW}_{0}$ and $\mathrm{GW}$ calculations, convergence has been achieved with a maximum error of $0.005 \mathrm{eV}$ for the eigenvalues. Here, we must warn that the procedure followed in the present work to carry out the $G_{0} W_{0}, G W_{0}$, or $G W$ calculations does not consider the update of the Kohn-Sham orbitals in a self-consistent way and only considers the update of the eigenvalues and of the corresponding Green's function. In principle, one could expect that updating the density would probably lead to a more accurate description, although results by Shishkin and Kresse ${ }^{12}$ show that this is not necessarily the case and that, in addition, these already computationally demanding approaches become almost unfeasible. Nevertheless, Shishkin and Kresse ${ }^{12}$ presented evidence that in case the starting, zero-order DFT result is qualitatively correct, it is enough to correct the eigenvalues for the quasiparticle effect. This is the procedure followed in the present work.

The PBE and different types of GW calculations have been carried out using the 5.2 version of the VASP code. ${ }^{32}$ For comparison, selected calculations for the ground-state electronic structure of $\mathrm{MgO}$ have been carried out with the B3LYP hybrid functional ${ }^{33,34}$ using the CRYSTAL09 code, ${ }^{35}$ and standard 8-411(d11)G and 8-511(d11)G Gaussian-type orbital basis sets for $\mathrm{O}$ and $\mathrm{Mg}$, respectively. ${ }^{36}$

\section{RESULTS AND DISCUSSION}

Electronic structure-related properties calculated for the three oxides are reported in Table I and compared with experimental values. ${ }^{37,38}$ First, one may notice that present PBE, $\mathrm{G}_{0} \mathrm{~W}_{0}, \mathrm{G}_{0} \mathrm{~W}$, and $\mathrm{GW}$ results for $\mathrm{ZnO}$ are in complete agreement with those reported earlier by Shishkin and Kresse, ${ }^{12}$ whereas those corresponding to $\mathrm{MgO}$ exhibit some slight differences. We have verified that the discrepancies in the $\mathrm{MgO}$ case arise from the use of slightly different unit cells: we use a PBE fully relaxed unit cell ( $\mathrm{a}=4.25 \AA$ ), whereas Shishkin and Kresse adopted the experimental lattice ( $\mathrm{a}=4.21 \AA$ ). A benchmark calculation with our setting and the experimental cell completely reproduced the results from Ref. 12 (PBE gives a band gap of $4.75 \mathrm{eV}$, compared to $4.76 \mathrm{eV}$ in Ref. 12, while $\mathrm{G}_{0} \mathrm{~W}_{0}, \mathrm{GW}_{0}$ and $\mathrm{GW}$ give 7.18, 7.64 and 8.37 $\mathrm{eV}$ respectively, in very good agreement with the $7.25,7.72$ and $8.47 \mathrm{eV}$ values reported in Ref. 12). In any case, the band gap predicted by the PBE functional largely underestimates the band gap of the three oxides, as expected. One might also

TABLE I. Calculated band gap, experimental excitation energies, and root mean square of the deviation from the experiment $(\mathrm{eV})$ for $\mathrm{MgO}, \mathrm{CaO}$, and $\mathrm{ZnO}$. Results in parenthesis from Shishkin and Kresse (Ref. 11).

\begin{tabular}{lccccc}
\hline \hline & PBE & $\mathrm{G}_{0} \mathrm{~W}_{0}$ & $\mathrm{GW}_{0}$ & $\mathrm{GW}$ & $\operatorname{Exp}$ \\
\hline $\mathrm{MgO}$ & $4.44(4.76)$ & $6.81(7.25)$ & $7.27(7.72)$ & $7.98(8.47)$ & $7.8^{\mathrm{a}}$ \\
$\mathrm{CaO}$ & 3.65 & 6.01 & 6.53 & 7.47 & $7.1^{\mathrm{b}}$ \\
$\mathrm{ZnO}$ & $0.73(0.67)$ & $2.18(2.12)$ & $2.56(2.54)$ & $3.22(3.20)$ & $3.3^{\mathrm{c}}$ \\
$\mathrm{RMS}$ & 3.1 & 1.0 & 0.6 & 0.3 & \\
\hline \hline
\end{tabular}

\section{${ }^{\mathrm{a}}$ Ref. 37.}

${ }^{\mathrm{b}}$ Ref. 37.

${ }^{\mathrm{c}}$ Ref. 38. 
TABLE II. Calculated electronic gap, experimental excitation energies, and root mean square of the deviation from the experiment $(\mathrm{eV})$ for $\mathrm{F}$ and $\mathrm{F}^{+}$centers in $\mathrm{MgO}, \mathrm{CaO}$, and $\mathrm{ZnO}$.

\begin{tabular}{llcclc}
\hline \hline & \multicolumn{5}{c}{ F center } \\
\hline & PBE & $\mathrm{G}_{0} \mathrm{~W}_{0}$ & $\mathrm{GW}_{0}$ & $\mathrm{GW}$ & Exp \\
$\mathrm{MgO}$ & 2.39 & 4.48 & 4.71 & 5.20 & $5.03^{\mathrm{a}}$ \\
$\mathrm{CaO}$ & 0.75 & 3.20 & 3.53 & 3.87 & $3.1^{\mathrm{b}}$ \\
$\mathrm{ZnO}$ & 1.15 & 2.29 & 2.52 & 2.96 & $2.45^{\mathrm{c}}$ \\
$\mathrm{RMS}$ & 2.2 & 0.3 & 0.3 & 0.5 & \\
& & & $\mathrm{~F}^{+}$center & & \\
& $\mathrm{PBE}$ & $\mathrm{G}_{0} \mathrm{~W}_{0}$ & $\mathrm{GW}_{0}$ & $\mathrm{GW}$ & $\mathrm{Exp}^{\mathrm{c}}$ \\
$\mathrm{MgO}$ & 2.95 & 5.12 & 5.40 & 5.90 & $4.96^{\mathrm{a}}$ \\
$\mathrm{CaO}$ & 1.93 & 4.55 & 4.96 & 5.62 & 3.7 \\
$\mathrm{RMS}$ & 1.9 & 0.6 & 0.9 & 1.5 & \\
\hline \hline
\end{tabular}

${ }^{\mathrm{a}}$ Ref. 23.

${ }^{\mathrm{b}}$ Ref. 39.

${ }^{c}$ Ref. 40 .

note that the explicit introduction of many-body effects on the calculated electron correlation results in calculated band-gap values that are closer to the experiment. More importantly, the root mean square error with respect to the experiment significantly decreases when improving the quality of the $\mathrm{GW}$ calculation. Obviously, this result has to be taken with care because only three cases are included in the statistics. Nevertheless, one should not be surprised by the fact that by improving the quality of the calculation, a better agreement with the experiment is achieved, which provides a genuine $a b$ initio flavor to this type of approach. We must also warn that the statement above is somehow different from the general conclusion of Shishkin and Kresse, ${ }^{12}$ who found the $\mathrm{GW}_{0}$ to generally provide better agreement with the experiment. For the sake of comparison, calculations of the band gap of bulk $\mathrm{MgO}$ have been carried out with the hybrid B3LYP functional; the calculated value of $7.15 \mathrm{eV}$ largely improves the GGA estimates, but it is still far from the GW predictions, which indeed nicely reproduce the experimental value.

Having verified that the GW approach is able to properly describe the electronic structure of bulk $\mathrm{MgO}, \mathrm{CaO}$, and $\mathrm{ZnO}$, we turn our attention to the oxygen vacancy-containing systems for which the resulting $\mathrm{F}$ and $\mathrm{F}^{+}$centers are well-characterized experimentally ${ }^{23,39-41}$ as well as by means of embedded cluster calculations with sophisticated configuration interaction wave functions. ${ }^{18-20}$ Table II summarizes the results for the lowest excitations of both $\mathrm{F}$ and $\mathrm{F}^{+}$centers as predicted from the various levels of theory considered in the present work. Overall, the different variants of the GW approach largely improve the prediction of PBE, which is indeed very poor. For $\mathrm{MgO}$, the most accurate $\mathrm{GW}$ value of $5.20 \mathrm{eV}$ compares very well with the experimental value of $5.03 \mathrm{eV}, 23$ and it is even better than the best estimate $(5.44 \mathrm{eV})$ from multireference second-order perturbation (CASPT2) calculations. ${ }^{18}$ This is a remarkable result, because it represents the best theoretical estimate, and it arises from a fully periodic method. Moreover, the calculated value is almost unaffected by the supercell size (defect concentration) because a similar value (5.19 eV) is obtained for the smaller $\mathrm{Mg}_{8} \mathrm{O}_{8}$ supercell. On this smaller unit cell, a B3LYP calculation has also been
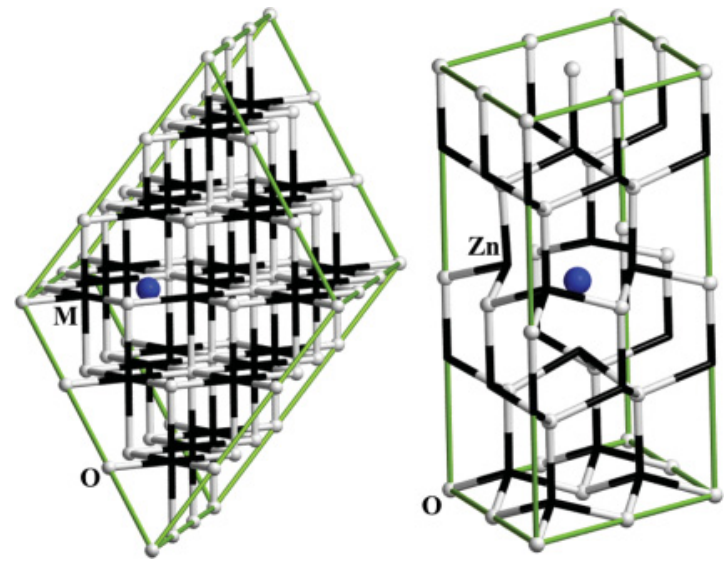

FIG. 1. (Color online) Representation of the supercells adopted in the calculations. $\mathrm{MgO}$ and $\mathrm{CaO}$ face-centered cubic (left) and $\mathrm{ZnO}$ Wurtzite (right). The huge sphere evidences the lattice position corresponding to the oxygen vacancy.

performed, giving a band gap of $4.22 \mathrm{eV}$ : as in the case of the perfect bulk, B3LYP largely improves the poor GGA result; nevertheless, it remarkably underestimates the F-center excitation.

A similar situation is found in the case of $\mathrm{ZnO}$, in which the best GW estimate is of $2.96 \mathrm{eV}$, only slightly larger than the $2.73 \mathrm{eV}$ predicted by the accurate multiconfiguration-coupled electron pair approximation (MCCEPA) calculations of Fink ${ }^{20}$ and close enough to the experimental value of $2.45 \mathrm{eV}^{40}$ A rather different situation is found for $\mathrm{CaO}$, where the GW-calculated F-center excitation is $0.8 \mathrm{eV}$ too high (3.87 versus $3.1 \mathrm{eV}$ ). The origin of this difference is the long-range response to the presence of the charged point defect. This is consistent with the findings of Carrasco et al. ${ }^{19}$ employing an embedded cluster model, which explicitly accounted for long-range polarization through the well-known shell model. Using time-dependent DFT within the B3LYP functional and a $\mathrm{Ca}_{14} \mathrm{O}_{13} \mathrm{O}_{\text {vac }}$ quantum mechanical cluster surrounded by 102 polarizable interface atoms, a value of $3.52 \mathrm{eV}$ is predicted, whereas CASPT2 calculations on a similar cluster yielded $4.18 \mathrm{eV}$. This interpretation is further supported by the fact that the GW band gap for our larger supercell $\left(\mathrm{Ca}_{27} \mathrm{O}_{26} \mathrm{O}_{\text {vac }}\right)$ differs from the one obtained for the smaller $\left(\mathrm{Ca}_{8} \mathrm{O}_{7} \mathrm{O}_{\text {vac }}\right)$ cell, whereas for the undefective systems the result obtained for the $\mathrm{Ca}_{27} \mathrm{O}_{27}$ and $\mathrm{Ca}_{8} \mathrm{O}_{8}$ cells is the same. In the case of $\mathrm{MgO}$, the energetic levels of the defective systems are not affected by the size of the supercell. Clearly, the difference between GW and experiment in $\mathrm{CaO}$ is not due to limitations in theory but on the use of a supercell, which in this case is still too small. Unfortunately, calculations with an even larger supercell are still too demanding.

Finally, we consider the $\mathrm{F}^{+}$centers involving only one trapped electrons and, hence, a charged supercell. In order to avoid artifacts arising from this charge, a compensating negative background charge has been employed in the calculations. Results in Table II show that in this case the agreement with experiment is not as good as in the case of the neutral $\mathrm{F}$ centers, although they all represent a significant improvement over the results obtained from the standard PBE band structure method. 


\section{CONCLUSIONS}

From the set of results reported in the previous section, one can firmly conclude that it is now possible to conjugate physically consistent models and good accuracy in the description of optical properties of oxygen vacancies in oxides and likely of other point defects in solids. This achievement opens the way to new studies concerning the simulation of defective insulators and semiconductors, even in those cases in which the use of embedded cluster models is controversial, such as $\mathrm{TiO}_{2}$ or $\mathrm{CeO}_{2}$, which are much less ionic, thus making the setup of an appropriate embedding scheme quite difficult.

The results in the present work show that quasiparticle periodic calculations with the GW method are able to provide an almost quantitative description of the $\mathrm{F}$ centers in $\mathrm{MgO}$,
$\mathrm{CaO}$, and $\mathrm{ZnO}$ and, more importantly, with an appropriate and physically meaningful solid-state model. The agreement is not as good for the charged $\mathrm{F}^{+}$centers, which is attributed to the need for an even larger supercell to properly describe the long-range electrostatic effect induced by this charge defect.

\section{ACKNOWLEDGMENTS}

This research has been supported by the Spanish MICINN through the program INNPACTO CASCADA Project No. IPT120000-2010-19 and research Grant No. FIS2008-02238 and, in part, by Generalitat de Catalunya Grants No. 2009SGR1041 and No. XRQTC. F.I. acknowledges additional support through the 2009 ICREA Academia Award for Excellence in Research. *francesc.illas@ub.edu

${ }^{1}$ J. G. Bednorz and K. A. Müller, Z. Phys. B 64, 189 (1986).

${ }^{2}$ S. Jin, T. H. Tiefel, M. McCormack, R. A. Fastnacht, R. Ramesh, and J. H. Chen, Science 264, 413 (1994).

${ }^{3}$ R. Asahi, T. Morikawa, T. Ohwaki, K. Aoki, and Y. Taga, Science 293, 269 (2001).

${ }^{4}$ E. Dagotto, Rev. Mod. Phys. 66, 763 (1994).

${ }^{5}$ W. E. Pickett, Rev. Mod. Phys. 61, 433 (1989).

${ }^{6}$ I. P. R. Moreira, F. Illas, and R. L. Martin, Phys. Rev. B 65, 155102 (2002).

${ }^{7}$ I. de P. R. Moreira, P. Rivero, and F. Illas, J. Chem. Phys. 134, 074709 (2011).

${ }^{8}$ P. Rivero, I. P. R. Moreira, and F. Illas, Phys. Rev. B 81, 205123(2010).

${ }^{9}$ F. Tran and P. Blaha, Phys. Rev. Lett. 102, 226401 (2009).

${ }^{10}$ L. Hedin, Phys. Rev. 139, A796 (1965).

${ }^{11}$ S. V. Faleev, M. van Schilfgaarde, and T. Kotani, Phys. Rev. Lett. 93, 126406 (2004).

${ }^{12}$ M. Shishkin and G. Kresse, Phys. Rev. B 75, 235102 (2007).

${ }^{13}$ M. Shishkin and G. Kresse, Phys. Rev. B 74, 035101 (2006).

${ }^{14}$ C. Rödl, F. Fuchs, J. Furthmüller, and F. Bechstedt, Phys. Rev. B 79, 235114 (2009).

${ }^{15}$ H. Jiang, R. I. Gomez-Abal, P. Rinke, and M. Scheffler, Phys. Rev. B 82, 045108 (2010).

${ }^{16}$ W. Kang and M. S. Hybertsen, Phys. Rev. B 82, 085203 (2010).

${ }^{17}$ M. Stoneham, Theories of Defects in Solids (Oxford University Press, Oxford, 2010).

${ }^{18}$ C. Sousa and F. Illas, J. Chem. Phys. 115, 1435 (2001).

${ }^{19}$ J. Carrasco, C. Sousa, F. Illas, P. V. Sushko, and A. L. Shluger, J. Chem. Phys. 125, 074710 (2006).

${ }^{20}$ K. Fink, Phys. Chem. Chem. Phys. 7, 2999 (2005).

${ }^{21}$ A. M. Ferrari and G. Pacchioni, J. Phys. Chem. 99, 17010 (1995).
${ }^{22}$ P. Mori-Sánchez, J. M. Recio, B. Silvi, C. Sousa, A. Martín Pendas,V. Luaña, and F. Illas, Phys. Rev. B 66, 075103 (2002).

${ }^{23}$ Y. Chen, R. T. Williams, and W. A. Sibley, Phys. Rev. 182, 960 (1969).

${ }^{24}$ G. Pacchioni, Chem. Phys. Chem. 4, 1041 (2003).

${ }^{25}$ L. Giordano and G. Pacchioni, Acc. Chem. Res. 44, 1244 (2011).

${ }^{26}$ J. P. Perdew, K. Burke, and M. Ernzerhof, Phys. Rev. Lett. 77, 3865 (1996).

${ }^{27}$ P. E. Blöchl, Phys. Rev. B 50, 17953 (1994).

${ }^{28}$ G. Kresse and D. Joubert, Phys. Rev. B 59, 1758 (1999).

${ }^{29}$ H. J. Monkhorst and J. D. Pack, Phys. Rev. B 13, 5188 (1976).

${ }^{30}$ F. Fuchs, J. Furthmüller, F. Bechstedt, M. Shishkin, and G. Kresse, Phys. Rev. B 76, 115109 (2007).

${ }^{31}$ M. Shishkin, M. Marsman, and G. Kresse, Phys. Rev. Lett. 99, 246403 (2007).

${ }^{32}$ G. Kresse and J. Furthmüller, J. Comput. Mater. Sci. 6, 15 (1996).

${ }^{33}$ A. D. Becke, J. Chem. Phys. 98, 5648 (1993).

${ }^{34}$ C. Lee, W. Yang, and R. G. Parr, Phys. Rev. B 37, 785 (1988).

${ }^{35}$ R. Dovesi, R. Orlando, B. Civalleri, C. Roetti, V. R. Saunders, and C. M. Zicovich-Wilson, Z. Kristallogr. 220, 571 (2005).

${ }^{36}$ L. Valenzano, Y. Noel, R. Orlando, C. M. Zicovich-Wilson, M. Ferrero, and R. Dovesi, Theor. Chem. Acc. 117, 991 (2006).

${ }^{37}$ R. C. Whited, C. J. Flaten, and W. C. Walker, Solid State Commun. 13, 1903 (1973).

${ }^{38}$ C. Kittel, Introduction to Solid State Physics, 6th ed. (Wiley, New York, 1986), p. 185.

${ }^{39}$ J. L. Park, Y. Chen, G. P. Williams, R. T. Williams, and G. J. Pogatshnik, Phys. Rev. B 43, 11991 (1991).

${ }^{40}$ K. Vanheusden, W. L. Warren, C. H. Seager, D. R. Tallant, J. A. Voigt, and B. E. Gnade, J. Appl. Phys. 79, 7983 (1996).

${ }^{41}$ J. M. Smith and W. E. Vehse, Phys. Lett. A 31, 147 (1970). 